\title{
High Order Projection Plane Method for Evaluation of Supersingular Curved Boundary Integrals in BEM
}

\author{
Miao Cui, Wei-zhe Feng, Xiao-wei Gao, and Kai Yang \\ School of Aeronautics and Astronautics, Faculty of Vehicle Engineering and Mechanics, State Key Laboratory of \\ Structural Analysis for Industrial Equipment, Dalian University of Technology, Dalian 116024, China
}

Correspondence should be addressed to Kai Yang; kyang@dlut.edu.cn

Received 7 December 2015; Revised 12 February 2016; Accepted 17 February 2016

Academic Editor: Ivano Benedetti

Copyright (C) 2016 Miao Cui et al. This is an open access article distributed under the Creative Commons Attribution License, which permits unrestricted use, distribution, and reproduction in any medium, provided the original work is properly cited.

Boundary element method (BEM) is a very promising approach for solving various engineering problems, in which accurate evaluation of boundary integrals is required. In the present work, the direct method for evaluating singular curved boundary integrals is developed by considering the third-order derivatives in the projection plane method when expanding the geometry quantities at the field point as Taylor series. New analytical formulas are derived for geometry quantities defined on the curved line/plane, and unified expressions are obtained for both two-dimensional and three-dimensional problems. For the twodimensional boundary integrals, analytical expressions for the third-order derivatives are derived and are employed to verify the complex-variable-differentiation method (CVDM) which is used to evaluate the high order derivatives for three-dimensional problems. A few numerical examples are given to show the effectiveness and the accuracy of the present method.

\section{Introduction}

Boundary element method (BEM) [1] is a very promising approach for solving various engineering problems, such as heat transfer problems [2-5] in energy science and engineering, in which accurate evaluation of boundary integrals [6-10] is required. Weak, strong, or hypersingularities are involved in these boundary integrals, if the source point is located on the element under integration [11]. These issues have been focused on by researchers, and many techniques, such as Gauss logarithmic quadrature rule for weak singularities [12], rigid body displacement algorithm for strong singularities [12], and regularization for hypersingularities [13], have been proposed to remove the singularities. Each method has its advantage and disadvantage [11-19]. A comprehensive review has been given in [11] on the treatment of singularities. In general, the efficient methods for singular curved line and surface integrals are those based on the operation over the intrinsic coordinate system [14-16].

Recently, Gao et al. [11] proposed another efficient direct method for numerically evaluating all kinds of singular curved boundary integrals for two-dimensional (2D) and three-dimensional (3D) BEM analyses, and numerical examples were given to demonstrate the stability of the proposed method. However, only quadratic terms were truncated when expanding the coordinate of the field point as Taylor series, which means the method will be invalid if the curved boundary is beyond the quadratic, such as the cubed curve.

In the present work, the direct method in [11] for evaluating arbitrary high order singular curved boundary integrals is developed, by considering the third-order derivatives when expanding the geometry quantities at the field point as Taylor series. Corresponding analytical expressions are derived. The first innovation is that the present method will extend the application range of the method in [11]. That is, the singular integrals over higher order elements, such as cubed curve, can be directly evaluated. Another innovation is that the complex-variable-differentiation method [20] is introduced to determine the third-order derivatives. For 2D boundary integral, the third-order derivatives are analytically derived, which are used to verify the CVDM.

The paper is organized as follows. In Section 2, review and modification of the direct method in [11] are provided. In Section 3, numerical examples are given to show the 
effectiveness and the accuracy of the present method. Finally, conclusions are drawn from computational results and discussions.

\section{Development of the Direct Method for Evaluating Arbitrary High Order Singular Curved Boundary Integrals}

2.1. Review of Singular Integrals [11]. Singular integrals over an element can be classified into the following form:

$$
I_{e}\left(\mathbf{x}^{p}\right)=\int_{\Gamma_{e}} \frac{\bar{f}\left(\mathbf{x}^{p}, \mathbf{x}\right)}{r^{\beta}\left(\mathbf{x}^{p}, \mathbf{x}\right)} d \Gamma(\mathbf{x})
$$

in which $\mathbf{x}^{p}$ and $\mathbf{x}$ represent the source and field points, respectively; $\Gamma_{e}$ is the boundary element under integration, which is a curved line or a curved surface for $2 \mathrm{D}$ or $3 \mathrm{D}$ problems, respectively; and $r$ is the distance between the source and field points.

The term $\bar{f}\left(\mathbf{x}^{p}, \mathbf{x}\right)$ in (1) is a regular function and $\beta$ is the order of the singularity.

In $2 \mathrm{D}$ problems, the following type of singular line integrals is also frequently encountered:

$$
J_{e}\left(\mathbf{x}^{p}\right)=\int_{\Gamma_{e}} \frac{\bar{f}\left(\mathbf{x}^{p}, \mathbf{x}\right) \ln \left[r\left(\mathbf{x}^{p}, \mathbf{x}\right)\right]}{r^{\beta}\left(\mathbf{x}^{p}, \mathbf{x}\right)} d \Gamma(\mathbf{x}) .
$$

It is assumed that the integrals in (1) and (2) always exist: that is, each integration has a finite value.

2.2. Review of Expansions of Geometry Quantities on the Projection Line/Plane [11]. In [11], a projection line for 2D or a projection plane for $3 \mathrm{D}$ problems, which is the tangential line/plane of the element to the origin of the intrinsic coordinate system, was introduced. A local orthogonal coordinate system was established on the projection line/plane. The coordinate transformation between the local and global systems can be performed using the following relationships:

$$
\begin{aligned}
& x_{i}^{\prime}=L_{i j}\left(x_{j}-x_{j}^{o}\right), \\
& x_{i}=x_{i}^{o}+L_{j i} x_{j}^{\prime},
\end{aligned}
$$

where $L_{i j}$ is the direction cosine of the local coordinate axes with respect to the global one. The repeated subscripts represent summation; $x_{i}^{o}$ is the global coordinates of the origin of the local coordinate system.

The original curved element can be projected onto the projection line or plane to form a straight or a flat projection element by (3), and then all geometry quantities can be expressed in terms of variables defined on the projection line/plane.

\subsection{High Order Expansion of Geometry Quantities in terms of} Variables on the Projection Line for 2D Problems. For 2D and 3D problems, derivations for the modification are different, which are separately described in detail.

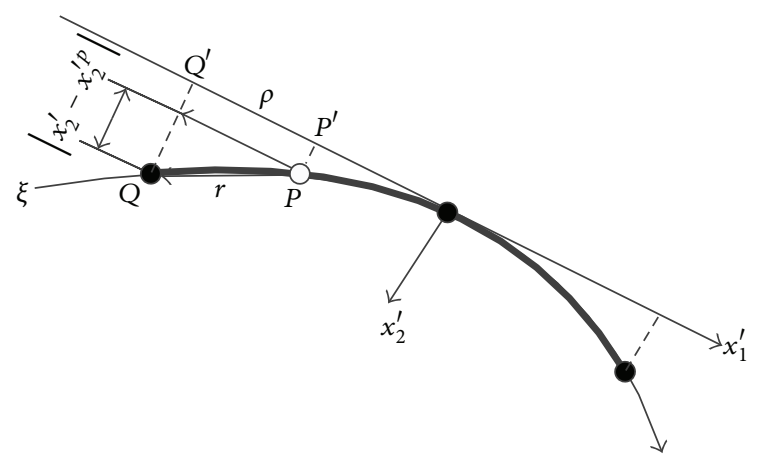

FIGURE 1: The curved line boundary and the projection line.

In $2 \mathrm{D}$ problems, the boundary element is a curved line as shown in Figure 1. The local orthogonal coordinate system is denoted by $\left(x_{1}^{\prime}, x_{2}^{\prime}\right)$ and the projection line is along the axis $x_{1}^{\prime}$.

There is only one independent variable for a line, and all other geometry quantities can be expressed by $x_{1}^{\prime}$. Similar to the treatment in [11], the coordinate $x_{2}^{\prime}$ at the field point over the curved line can be expressed by $x_{1}^{\prime}$ using Taylor series. Different from the treatment in [11], the third-order derivative is considered in the present work:

$$
\begin{aligned}
x_{2}^{\prime} & =\frac{\partial x_{2}^{\prime}}{\partial x_{1}^{\prime}} x_{1}^{\prime}+\frac{1}{2} \frac{\partial^{2} x_{2}^{\prime}}{\partial x_{1}^{\prime 2}} x_{1}^{\prime 2}+\frac{1}{6} \frac{\partial^{3} x_{2}^{\prime}}{\partial x_{1}^{\prime 3}} x_{1}^{\prime 3} \\
& =a_{1}\left(x_{2}^{\prime}\right) x_{1}^{\prime}+a_{2}\left(x_{2}^{\prime}\right) x_{1}^{\prime 2}+a_{3}\left(x_{2}^{\prime}\right) x_{1}^{\prime 3},
\end{aligned}
$$

where

$$
\begin{aligned}
& a_{1}\left(x_{2}^{\prime}\right)=\frac{\partial x_{2}^{\prime}}{\partial x_{1}^{\prime}}=\frac{\partial x_{2}^{\prime}}{\partial \xi_{1}} \frac{\partial \xi_{1}}{\partial x_{1}^{\prime}} \\
& a_{2}\left(x_{2}^{\prime}\right)=\frac{1}{2} \frac{\partial^{2} x_{2}^{\prime}}{\partial x_{1}^{\prime 2}}=\frac{1}{2}\left\{\frac{\partial^{2} x_{2}^{\prime}}{\partial \xi^{2}} \frac{\partial \xi}{\partial x_{1}^{\prime}} \frac{\partial \xi}{\partial x_{1}^{\prime}}+\frac{\partial x_{2}^{\prime}}{\partial \xi} \frac{\partial^{2} \xi}{\partial x_{1}^{\prime 2}}\right\} \\
& a_{3}\left(x_{2}^{\prime}\right)=\frac{1}{6} \frac{\partial^{3} x_{2}^{\prime}}{\partial x_{1}^{\prime 3}}=\frac{1}{6} \frac{\partial}{\partial x_{1}^{\prime}}\left(\frac{\partial^{2} x_{2}^{\prime}}{\partial x_{1}^{\prime 2}}\right) \\
& =\frac{1}{6}\left\{\frac{\partial^{3} x_{2}^{\prime}}{\partial \xi^{3}} \frac{\partial \xi}{\partial x_{1}^{\prime}} \frac{\partial \xi}{\partial x_{1}^{\prime}} \frac{\partial \xi}{\partial x_{1}^{\prime}}+3 \frac{\partial^{2} x_{2}^{\prime}}{\partial \xi^{2}} \frac{\partial \xi}{\partial x_{1}^{\prime}} \frac{\partial^{2} \xi}{\partial x_{1}^{\prime 2}}\right. \\
& \left.+\frac{\partial x_{2}^{\prime}}{\partial \xi} \frac{\partial^{3} \xi}{\partial x_{1}^{\prime 3}}\right\} \cdot
\end{aligned}
$$

It can be seen that the expressions are more complex. For $2 \mathrm{D}$ problem, the corresponding derivatives are analytically derived, and the detailed derivation process is omitted here. 
In (5) and (6),

$$
\begin{aligned}
\frac{\partial x_{2}^{\prime}}{\partial \xi} & =\sum_{\alpha=1}^{N_{\text {node }}} \frac{\partial N_{\alpha}}{\partial \xi} x_{2}^{\prime \alpha}, \\
\frac{\partial^{2} x_{2}^{\prime}}{\partial \xi^{2}} & =\sum_{\alpha=1}^{N_{\text {node }}} \frac{\partial^{2} N_{\alpha}}{\partial \xi^{2}} x_{2}^{\prime \alpha}, \\
\frac{\partial^{3} x_{2}^{\prime}}{\partial \xi^{3}} & =\sum_{\alpha=1}^{N_{\text {node }}} \frac{\partial^{3} N_{n}}{\partial \xi^{3}} x_{2}^{\prime \alpha}, \\
\frac{\partial \xi}{\partial x_{1}^{\prime}} & =\frac{1}{\left|m_{1}\right|}, \\
\frac{\partial^{2} \xi}{\partial x_{1}^{\prime 2}} & =-\frac{1}{\left|m_{1}\right|^{4}}\left(\frac{\partial x_{1}}{\partial \xi} \frac{\partial^{2} x_{1}}{\partial \xi^{2}}+\frac{\partial x_{2}}{\partial \xi} \frac{\partial^{2} x_{2}}{\partial \xi^{2}}\right), \\
\frac{\partial^{3} \xi}{\partial x_{1}^{\prime 3}} & =\frac{\partial}{\partial x_{1}^{\prime}}\left(\frac{\partial^{2} \xi}{\partial x_{1}^{\prime 2}}\right)=-\frac{m_{1}^{2} B-4 C}{m_{1}{ }^{7}} .
\end{aligned}
$$

In (7a)-(8c),

$$
\begin{aligned}
\left|m_{1}\right|= & \sqrt{\left(\frac{\partial x_{1}}{\partial \xi}\right)^{2}+\left(\frac{\partial x_{2}}{\partial \xi}\right)^{2}}, \\
B= & \left(\frac{\partial^{2} x_{1}}{\partial \xi^{2}}\right)^{2}+\left(\frac{\partial^{2} x_{2}}{\partial \xi^{2}}\right)^{2}+\frac{\partial x_{1}}{\partial \xi} \frac{\partial^{3} x_{1}}{\partial \xi^{3}} \\
& +\frac{\partial x_{2}}{\partial \xi} \frac{\partial^{3} x_{2}}{\partial \xi^{3}} \\
C= & \left(\frac{\partial x_{1}}{\partial \xi}\right)^{2}\left(\frac{\partial^{2} x_{1}}{\partial \xi^{2}}\right)^{2}+2 \frac{\partial x_{1}}{\partial \xi} \frac{\partial x_{2}}{\partial \xi} \frac{\partial^{2} x_{1}}{\partial \xi^{2}} \frac{\partial^{2} x_{2}}{\partial \xi^{2}} \\
& +\left(\frac{\partial x_{2}}{\partial \xi}\right)^{2}\left(\frac{\partial^{2} x_{2}}{\partial \xi^{2}}\right)^{2} .
\end{aligned}
$$

Define a local distance:

$$
\rho=\left\|x_{1}^{\prime}-x_{1}^{\prime p}\right\|,
$$

where $x_{1}^{\prime p}$ is the local coordinate of point $P^{\prime}$ which is the projection point of the source point $P$ and $x_{1}^{\prime}$ is the local coordinate of point $Q^{\prime}$ which is the projection point of the field point $Q$.

The derivative of $\rho$ with respect to local coordinate $x_{1}^{\prime}$ is

$$
\rho_{, 1}=\frac{\partial \rho}{\partial x_{1}^{\prime}}=\frac{x_{1}^{\prime}-x_{1}^{\prime p}}{\rho} .
$$

In (12a), $\rho_{, 1}=1$ or -1 .

Then, we can obtain

$$
x_{1}^{\prime}=x_{1}^{\prime p}+\rho_{, 1} \rho .
$$

By substituting (12a) into (5), we can obtain

$$
x_{2}^{\prime}=p\left(x_{2}^{\prime}\right)+q\left(x_{2}^{\prime}, \rho\right) \rho,
$$

where

$$
\begin{aligned}
p\left(x_{2}^{\prime}\right)= & a_{1} x_{1}^{\prime p}+a_{2}\left(x_{2}^{\prime}\right) x_{1}^{\prime p} x_{1}^{\prime p} \\
& +a_{3}\left(x_{2}^{\prime}\right) x_{1}^{\prime p} x_{1}^{\prime p} x_{1}^{\prime p}=x_{2}^{\prime p}, \\
q\left(x_{2}^{\prime}, \rho\right)= & q_{1}\left(x_{2}^{\prime}\right)+q_{2}\left(x_{2}^{\prime}\right) \rho+q_{3}\left(x_{2}^{\prime}\right) \rho^{2} .
\end{aligned}
$$

In (15),

$$
\begin{aligned}
& q_{1}\left(x_{2}^{\prime}\right) \\
& \quad=\left[a_{1}\left(x_{2}^{\prime}\right)+2 a_{2}\left(x_{2}^{\prime}\right) x_{1}^{\prime p}+3 a_{3}\left(x_{2}^{\prime}\right) x_{1}^{\prime p 2}\right] \rho_{, 1}, \\
& q_{2}\left(x_{2}^{\prime}\right)=a_{2}\left(x_{2}^{\prime}\right) \rho_{, 1}^{2}+3 a_{3}\left(x_{2}^{\prime}\right) x_{1}^{\prime p} \\
& \quad=a_{2}\left(x_{2}^{\prime}\right)+3 a_{3}\left(x_{2}^{\prime}\right) x_{1}^{\prime p}, \\
& q_{3}\left(x_{2}^{\prime}, \rho\right)=a_{3}\left(x_{2}^{\prime}\right) \rho_{, 1} .
\end{aligned}
$$

Similarly, we can express the intrinsic coordinate in terms of the local distance as

$$
\xi=p(\xi)+q(\xi, \rho) \rho,
$$

where $p(\xi)$ and $q(\xi, \rho)$ can be determined by replacing $x_{2}^{\prime}$ with $\xi$ in (13)-(16c).

Substituting (12b) and (13) into (4), we can obtain

$$
\begin{aligned}
x_{i} & =x_{i}^{o}+L_{j i} x_{j}^{\prime}=x_{i}^{o}+L_{1 i} x_{1}^{\prime}+L_{2 i} x_{2}^{\prime} \\
& =x_{i}^{o}+L_{1 i}\left(x_{1}^{\prime p}+\rho_{, 1} \rho\right)+L_{2 i}\left[x_{2}^{\prime p}+q\left(x_{2}^{\prime}, \rho\right) \rho\right] \\
& =x_{i}^{p}+L_{1 i} \rho_{, 1} \rho+L_{2 i} q\left(x_{2}^{\prime}, \rho\right) \rho .
\end{aligned}
$$

Then, substituting (15) into (18), we can obtain

$$
x_{i}=x_{i}^{p}+\left(b_{i}+c_{i} \rho+d_{i} \rho^{2}\right) \rho,
$$

where

$$
\begin{aligned}
b_{i} & =L_{1 i} \rho_{, 1}+L_{2 i} q_{1}\left(y^{\prime}\right), \\
c_{i} & =L_{2 i} q_{2}\left(y^{\prime}\right), \\
d_{i} & =L_{2 i} q_{3}\left(y^{\prime}\right) .
\end{aligned}
$$

Referring to Figure 1 , the global distance $r$ can be easily expressed as

$$
r=\sqrt{\left(x_{i}-x_{i}^{p}\right)\left(x_{i}-x_{i}^{p}\right)}=g(\rho) \rho,
$$

where

$$
\begin{aligned}
g(\rho) & =\sqrt{\left(b_{i}+c_{i} \rho+d_{i} \rho^{2}\right)\left(b_{i}+c_{i} \rho+d_{i} \rho^{2}\right)} \\
& =\sqrt{G_{0}+G_{1} \rho+G_{2} \rho^{2}+G_{3} \rho^{3}+G_{4} \rho^{4}} .
\end{aligned}
$$

In (22), $G_{0}=b_{i} b_{i}, G_{1}=2 b_{i} c_{i}, G_{2}=c_{i} c_{i}+2 b_{i} d_{i}, G_{3}=2 c_{i} d_{i}$, and $G_{4}=d_{i} d_{i}$.

It can be easily derived that

$$
r_{, i}=\frac{\partial r}{\partial x_{i}}=\frac{x_{i}-x_{i}^{p}}{r}=\frac{b_{i}+c_{i} \rho+d_{i} \rho^{2}}{g(\rho)} .
$$


2.4. The Complex-Variable-Differentiation Method (CVDM). From the above derivations, it can be seen that the corresponding expressions have been changed, by considering the third-order derivatives when expanding the geometry quantities at the field point as Taylor series. The third-order derivatives should be first determined.

In the present work, the complex-variable-differentiation method (CVDM) [20] is introduced to determine the high order derivatives. For $2 \mathrm{D}$ analysis, the third-order derivatives are determined explicitly by (7c), (8c), (9), (10a), and (10b), which are also calculated by the CVDM.

In the CVDM, the variable $x$ of a real function $f(x)$ is replaced by a complex one $x+i h$, with the imaginary part $h$ being very small (usually $h=10^{-20}$ ). The function $f(x+i h)$ can be expanded into Taylor's series as

$$
f(x+i h)=f(x)+i h f^{\prime}(x)-\frac{h^{2}}{2} f^{\prime \prime}(x)+o\left(h^{3}\right) .
$$

Since $h$ is small enough, (25) can be obtained:

$$
f^{\prime}(x)=\frac{\operatorname{Im}(f(x+i h))}{h}
$$

where Im denotes the imaginary part.

The CVDM is a very promising method, since the derivatives only require direct evaluations [21]. The CVDM has been validated to be an efficient and accurate approach for determination of derivatives [22-24].

For example, considering $\partial^{3} x_{2}^{\prime} / \partial x_{1}^{\prime 3}=\left(\partial / \partial x_{1}^{\prime}\right)\left(\partial^{2} x_{2}^{\prime} /\right.$ $\left.\partial x_{1}^{\prime 2}\right)$, we can add the very small imaginary part $h$ to $x_{1}^{\prime}$; then the real value of $\partial^{3} x_{2}^{\prime} / \partial x_{1}^{\prime 3}$ yields $\operatorname{Im}\left(\partial^{2} x_{2}^{\prime} / \partial x_{1}^{\prime 2}\right) / h$. In addition, the value of $\partial^{2} x_{2}^{\prime} / \partial x_{1}^{\prime 2}$ can be easily obtained using the analytical expressions in the previous work [11]. The CVDM and the derived expressions in (7c)-(8c) can be validated by each other.

2.5. Expansion of Geometry Quantities in terms of Variables on the Projection Plane for 3D Problems. In 3D problems, the boundary element is a curved surface marked as $A B C D$ in Figure 2. A local orthogonal coordinate system $\left(x_{1}^{\prime}, x_{2}^{\prime}, x_{3}^{\prime}\right)$ is established on the projection plane with its origin being at the point $\left(\xi_{1}=0, \xi_{2}=0\right)$, in which axes $x_{1}^{\prime}$ and $x_{2}^{\prime}$ are located within the plane. The axis $x_{1}^{\prime}$ is along $\xi_{1}$ direction and axis $x_{3}^{\prime}$ is along the outward normal direction to the element. By (4), we can project the original curved surface element onto the projection plane, to form a flat projection element marked as $A^{\prime} B^{\prime} C^{\prime} D^{\prime}$ in Figure 2.

In a plane, there are only two independent variables. We choose $x_{1}^{\prime}$ and $x_{2}^{\prime}$ as the independent variables, and $x_{3}^{\prime}$ over the curved surface can be expressed in terms of $x_{1}^{\prime}$ and $x_{2}^{\prime}$. Compared with the treatment in [11], $x_{3}^{\prime}$ is expanded as Taylor series by $x_{1}^{\prime}$ and $x_{2}^{\prime}$, by considering the third-order derivatives:

$$
x_{3}^{\prime}=a_{I}\left(x_{3}^{\prime}\right) x_{I}^{\prime}+a_{I J}\left(x_{3}^{\prime}\right) x_{I}^{\prime} x_{J}^{\prime}+a_{I J K}\left(x_{3}^{\prime}\right) x_{I}^{\prime} x_{J}^{\prime} x_{K}^{\prime},
$$

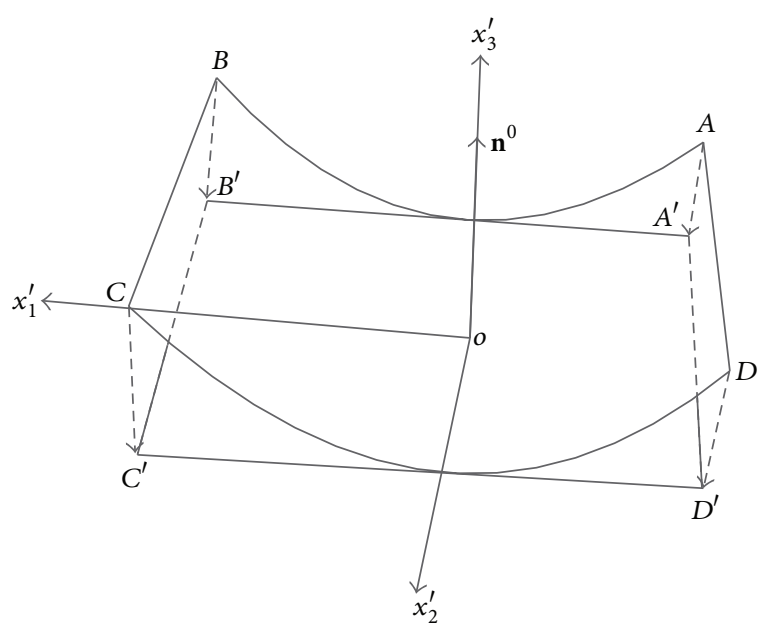

FIGURE 2: The curved surface boundary and the projection plane.

where $I, J$, and $K$ are integers varying from 1 to 2 , and

$$
\begin{aligned}
a_{I}\left(x_{3}^{\prime}\right) & =\frac{\partial x_{3}^{\prime}}{\partial x_{I}^{\prime}}=\frac{\partial x_{3}^{\prime}}{\partial \xi_{K}} \frac{\partial \xi_{K}}{\partial x_{I}^{\prime}}, \\
a_{I J}\left(x_{3}^{\prime}\right) & =\frac{1}{2} \frac{\partial^{2} x_{3}^{\prime}}{\partial x_{I}^{\prime} \partial x_{J}^{\prime}} \\
& =\frac{1}{2}\left\{\frac{\partial^{2} x_{3}^{\prime}}{\partial \xi_{K} \partial \xi_{L}} \frac{\partial \xi_{K}}{\partial x_{I}^{\prime}} \frac{\partial \xi_{L}}{\partial x_{J}^{\prime}}+\frac{\partial x_{3}^{\prime}}{\partial \xi_{K}} \frac{\partial^{2} \xi_{K}}{\partial x_{I}^{\prime} \partial x_{J}^{\prime}}\right\}, \\
a_{I J K}\left(x_{3}^{\prime}\right) & =\frac{1}{6} \frac{\partial^{3} x_{3}^{\prime}}{\partial x_{I}^{\prime} \partial x_{J}^{\prime} \partial x_{K}^{\prime}}=\frac{1}{3} \frac{\partial a_{I J}\left(x_{3}^{\prime}\right)}{\partial x_{K}^{\prime}} \\
& =\frac{1}{3} \frac{\partial a_{I J}\left(x_{3}^{\prime}\right)}{\partial \xi_{L}} \frac{\partial \xi_{L}}{\partial x_{K}^{\prime}} .
\end{aligned}
$$

The treatment is the same as that in [11], to determine $a_{I}\left(x_{3}^{\prime}\right)$ in $(27 \mathrm{a})$ and $a_{I J}\left(x_{3}^{\prime}\right)$ in (27b). For $a_{I J K}\left(x_{3}^{\prime}\right)$ in $(27 \mathrm{c})$, we use the CVDM, to avoid rather complicated analytical derivations.

The local distance $\rho$ projected from the global distance $r$ onto the projection plane and its derivative $\rho_{, I}$ are introduced as follows:

$$
\begin{aligned}
\rho & =\sqrt{\left(x_{1}^{\prime}-x_{1}^{\prime p}\right)^{2}+\left(x_{2}^{\prime}-x_{2}^{\prime p}\right)^{2}}, \\
\rho_{, I} & =\frac{\partial \rho}{\partial x_{I}^{\prime}}=\frac{x_{I}^{\prime}-x_{I}^{\prime p}}{\rho} .
\end{aligned}
$$

The local coordinates at the field point can be expressed as

$$
x_{I}^{\prime}=x_{I}^{\prime p}+\rho_{, I} \rho .
$$

Similar to the 2D analysis, substituting (29) into (26) yields

$$
x_{3}^{\prime}=p\left(x_{3}^{\prime}\right)+q\left(x_{3}^{\prime}, \rho\right) \rho
$$


where

$$
\begin{aligned}
p\left(x_{3}^{\prime}\right)= & a_{I}\left(x_{3}^{\prime}\right) x_{I}^{\prime p}+a_{I J}\left(x_{3}^{\prime}\right) x_{I}^{\prime p} x_{J}^{\prime p} \\
& +a_{I J K}\left(x_{3}^{\prime}\right) x_{I}^{\prime p} x_{J}^{\prime p} x_{K}^{\prime p}=x_{3}^{\prime p}, \\
q\left(x_{3}^{\prime}, \rho\right)= & q_{1}\left(x_{3}^{\prime}\right)+q_{2}\left(x_{3}^{\prime}\right) \rho+q_{3}\left(x_{3}^{\prime}\right) \rho^{2} .
\end{aligned}
$$

In (32),

$$
\begin{aligned}
q_{1}\left(x_{3}^{\prime}\right)= & a_{I}\left(x_{3}^{\prime}\right) \rho_{, I}+a_{I J}\left(x_{3}^{\prime}\right) x_{I}^{\prime p} \rho_{, J} \\
& +a_{I J}\left(x_{3}^{\prime}\right) x_{J}^{\prime p} \rho_{, I}+a_{I J K}\left(x_{3}^{\prime}\right) x_{I}^{\prime p} x_{K}^{\prime p} \rho_{, J} \\
& +a_{I J K}\left(x_{3}^{\prime}\right) x_{J}^{\prime p} x_{K}^{\prime p} \rho_{, I} \\
& +a_{I J K}\left(x_{3}^{\prime}\right) x_{I}^{\prime p} x_{J}^{\prime p} \rho_{, K}, \\
q_{2}\left(x_{3}^{\prime}\right)= & a_{I J}\left(x_{3}^{\prime}\right) \rho_{, I} \rho_{, J}+a_{I J K}\left(x_{3}^{\prime}\right) x_{K}^{\prime p} \rho_{, I} \rho_{, J} \\
& +a_{I J K}\left(x_{3}^{\prime}\right) x_{I}^{\prime p} \rho_{, J} \rho_{, K} \\
& +a_{I J K}\left(x_{3}^{\prime}\right) x_{J}^{\prime p} \rho_{, I} \rho_{, K}, \\
q_{3}\left(x_{3}^{\prime}\right)= & a_{I J K}\left(x_{3}^{\prime}\right) \rho_{, I} \rho_{, J} \rho_{, K} .
\end{aligned}
$$

Similar to the 2D analysis, intrinsic coordinates can be expressed as

$$
\xi_{K}=p\left(\xi_{K}\right)+q\left(\xi_{K}, \rho\right) \rho
$$

where $p\left(\xi_{K}\right)$ and $q\left(\xi_{K}, \rho\right)$ can be determined by replacing $x_{3}^{\prime}$ with $\xi_{K}$ in (31)-(33c).

Substituting (29) and (30) into (4) yields

$$
\begin{aligned}
x_{i} & =x_{i}^{o}+L_{J i} x_{J}^{\prime}+L_{3 i} x_{3}^{\prime} \\
& =x_{i}^{o}+L_{J i}\left(x_{J}^{\prime p}+\rho_{, J} \rho\right)+L_{3 i}\left[x_{3}^{\prime p}+q\left(x_{3}^{\prime}, \rho\right) \rho\right] \\
& =x_{i}^{p}+L_{J i} \rho_{, J} \rho+L_{3 i} q\left(x_{3}^{\prime}, \rho\right) \rho .
\end{aligned}
$$

Substituting (32) into (35), we can obtain

$$
x_{i}=x_{i}^{p}+\left(b_{i}+c_{i} \rho+d_{i} \rho^{2}\right) \rho,
$$

where

$$
\begin{aligned}
& b_{i}=L_{J i} \rho_{, J}+L_{3 i} q_{1}\left(x_{3}^{\prime}\right), \\
& c_{i}=L_{3 i} q_{2}\left(x_{3}^{\prime}\right), \\
& d_{i}=L_{3 i} q_{3}\left(x_{3}^{\prime}\right) .
\end{aligned}
$$

The expression of $r$ is as follows:

$$
r=\sqrt{\left(x_{i}-x_{i}^{p}\right)\left(x_{i}-x_{i}^{p}\right)}=g(\rho) \rho,
$$

where

$$
\begin{aligned}
g(\rho) & =\sqrt{\left(b_{i}+c_{i} \rho+d_{i} \rho^{2}\right)\left(b_{i}+c_{i} \rho+d_{i} \rho^{2}\right)} \\
& =\sqrt{G_{0}+G_{1} \rho+G_{2} \rho^{2}+G_{3} \rho^{3}+G_{4} \rho^{4}} .
\end{aligned}
$$

In (39), $G_{0}=b_{i} b_{i}, G_{1}=2 b_{i} c_{i}, G_{2}=c_{i} c_{i}+2 b_{i} d_{i}, G_{3}=2 c_{i} d_{i}$, and $G_{4}=d_{i} d_{i}$.

It can be easily derived that $r_{, i}$ has the same expression as that in $2 \mathrm{D}$ analysis.

Considering that $I=J=K=1$ and $\rho_{, 1}=1$ or -1 for $2 \mathrm{D}$ problems, it can be seen that unified expressions are obtained for both two-dimensional and three-dimensional problems.

Then, we can handle the singular boundary integrals in (1) and (2), with the above modifications. The $2 \mathrm{D}$ problem is taken as an example to describe the procedure to determine the integral in (1). The differential relationship between the local distance and the real curved line can be written as

$$
d \rho=d \Gamma \cos \phi=d \Gamma n_{i}^{0} n_{i}
$$

where $n_{i}^{0}$ and $n_{i}$ are the outward normals to the tangential lines of passing the origin of the local coordinate system and the field point, respectively, and $\phi$ is the angle between the two outward normals.

Substituting (40) and (38) into (1) leads to

$$
I_{e}\left(\mathbf{x}^{p}\right)=\lim _{\varepsilon \rightarrow 0} \int_{\varepsilon}^{\rho_{E}} \frac{\bar{F}(\rho)}{\rho^{\beta}} d \rho,
$$

where $\rho_{E}$ is the distance from the projected source point $P^{\prime}$ to one of the ends of the projected line element and $\bar{F}(\rho)$ is the regular part: that is,

$$
\bar{F}(\rho)=\frac{\bar{f}\left(\mathbf{x}^{p}, \mathbf{x}(\rho)\right)}{g^{\beta}(\rho) n_{i}^{0} n_{i}(\rho)} .
$$

In order to integrate (41), the nonsingular part $\bar{F}$ is expanded as a power series in $\rho$, such that

$$
\bar{F}(\rho)=\sum_{n=0}^{N} B^{(n)} \rho^{n}
$$

in which $N$ is the order of the power series, usually taking a value between 2 and 7 depending on the size of $\rho_{E}$, and $B^{(n)}$ are constants which are determined by collocating $N+1$ points over the integration region $\left(0, \rho_{E}\right)$. In the paper, $N+1$ equally spaced points are used: that is, $\left(0, \rho_{1}, \ldots, \rho_{N}\right)$. The coefficient for the first point $(n=0)$ is $B^{(0)}=\bar{F}(0)$ and other coefficients can be solved using the following equation set:

$$
[R]\{B\}=\{Y\},
$$

where $[R]$ is a square matrix with the order of $N$ :

$$
[R]=\left[\begin{array}{cccc}
1 & \rho_{1} & \cdots & \rho_{1}^{N-1} \\
1 & \rho_{2} & \cdots & \rho_{2}^{N-1} \\
\vdots & \vdots & \cdots & \vdots \\
1 & \rho_{N} & \cdots & \rho_{N}^{N-1}
\end{array}\right]
$$


$\{B\}$ and $\{Y\}$ are vectors as follows:

$$
\begin{aligned}
& \{B\}=\left\{\begin{array}{c}
B^{1} \\
B^{2} \\
\vdots \\
B^{N}
\end{array}\right\}, \\
& \{Y\}=\left\{\begin{array}{c}
\frac{\left[\bar{F}\left(\rho_{1}\right)-B^{(0)}\right]}{\rho_{1}} \\
\frac{\left[\bar{F}\left(\rho_{2}\right)-B^{(0)}\right]}{\rho_{2}} \\
\vdots \\
\frac{\left[\bar{F}\left(\rho_{N}\right)-B^{(0)}\right]}{\rho_{N}}
\end{array}\right\} .
\end{aligned}
$$

Solving (44) for coefficient vector $\{B\}$ and then substituting (43) into (41) yield

$$
I_{e}\left(\mathbf{x}^{p}\right)=\sum_{n=0}^{N} B^{(n)} \lim _{\varepsilon \rightarrow 0} \int_{\mathcal{\varepsilon}}^{\rho_{E}} \rho^{n-\beta} d \rho=\sum_{n=0}^{N} B^{(n)} E_{n},
$$

where

$$
\begin{aligned}
& E_{n} \\
& = \begin{cases}\frac{1}{n-\beta+1}\left(\frac{1}{\rho_{E}^{\beta-n-1}}-\lim _{\rho_{\varepsilon} \rightarrow 0} \frac{1}{\rho_{\varepsilon}^{\beta-n-1}}\right) & (n \neq \beta-1) \\
\ln \rho_{E}-\lim _{\rho_{\varepsilon} \rightarrow 0} \ln \rho_{\varepsilon} & (n=\beta-1) .\end{cases}
\end{aligned}
$$

Then, singularities can be analytically removed.

\section{Numerical Examples}

Based on the Fortran code for singular integrals in [11], a new Fortran code has been developed with the above modification presented in this paper. It should be emphasized that the functions of the old Fortran code are fully included in the new Fortran code. If all the third-order derivatives are set to be zero, the functions are the same with those of the method in [11]. Besides, the new code has its unique characteristics.

For 2D analysis, the third-order derivatives are computed by both CVDM and analytical expression. For 3D analysis, the high order derivatives are computed by the CVDM, because analytical derivations are very difficult and complicated to be obtained.

In this section, the first example is given to show the advantage of CVDM over a difference method. Then, two singular integrals are calculated, to show the effectiveness and the accuracy of the present method.

3.1. The Advantage of the CVDM. A singular function is selected:

$$
f\left(x, x^{p}\right)=\frac{1}{\left(x-x^{p}\right)^{2}}
$$

TABLE 1: The first-order derivatives with different $h$ for $f\left(x, x^{p}\right)=$ $1 /\left(x-x^{p}\right)^{2}$.

\begin{tabular}{lcc}
\hline$h$ & CVDM & Difference method \\
\hline $1.00 E-04$ & $1.9603210 E+09$ & $2.0403182 E+09$ \\
$1.00 E-06$ & $1.9997157 E+09$ & $1.9997236 E+09$ \\
$1.00 E-08$ & $1.9997197 E+09$ & $1.9997197 E+09$ \\
$1.00 E-10$ & $1.9997197 E+09$ & $1.9997194 E+09$ \\
$1.00 E-12$ & $1.9997197 E+09$ & $1.9997857 E+09$ \\
$1.00 E-14$ & $1.9997197 E+09$ & $1.9981112 E+09$ \\
$1.00 E-16$ & $1.9997197 E+09$ & $1.1100197 E+09$ \\
$1.00 E-17$ & $1.9997197 E+09$ & $0.0000000 E+00$ \\
$1.00 E-28$ & $1.9997197 E+09$ & $0.0000000 E+00$ \\
\hline
\end{tabular}

Then, the exact derivative with respect to the variable $x^{p}$ can be obtained:

$$
\frac{\partial f}{\partial x^{p}}=\frac{2}{\left(x-x^{p}\right)^{3}} .
$$

For comparison, the central difference formulation is adopted: that is,

$$
\frac{\partial f}{\partial x^{p}}=\frac{f\left(x, x^{p}+h\right)-f\left(x, x^{p}-h\right)}{2 h} .
$$

When $x=1.001, x^{p}=1$, the exact first-order derivative is $2 \times 10^{9}$. The dependence of the derivatives on $h$ for the CVDM and the difference method is examined, and the results are listed in Table 1.

It can be seen that the difference method can give satisfactory results only for $h$ between $10^{-5}$ and $10^{-10}$. For smaller $h$, its result becomes unacceptable. However, the result of the CVDM tends to be independent of $h$ as the step size becomes sufficiently small. This clearly verifies the advantage of the CVDM.

3.2. A Weakly Singular Line Integral over a Cubed Curve. In this example, a singular line integral for a 2D BEM problem is chosen:

$$
J=\int_{\Gamma} \ln (r) d \Gamma
$$

The integration line is a cubed curve as shown in Figure 3.

The four typical points are $1(-1,-1), 2(1,1), 3$ $(-1 / 3,-1 / 27)$, and $4(1 / 3,1 / 27)$. The computational point (source point) is at 1 . The curve is taken as one boundary element. It should be emphasized here that the direct method in [11] has been invalid, which cannot be used for the singular line integration in (52) over the cubed curve in Figure 4, with only one element, because only quadratic terms were truncated when expanding the geometry quantities at the field point as Taylor series.

Firstly, the accuracy of the CVDM for the proposed technique is validated. We use the derived analytical expressions in $(7 c)-(8 c)$ to compute the third-order derivatives. Then, the CVDM is employed to determine the same derivatives. 


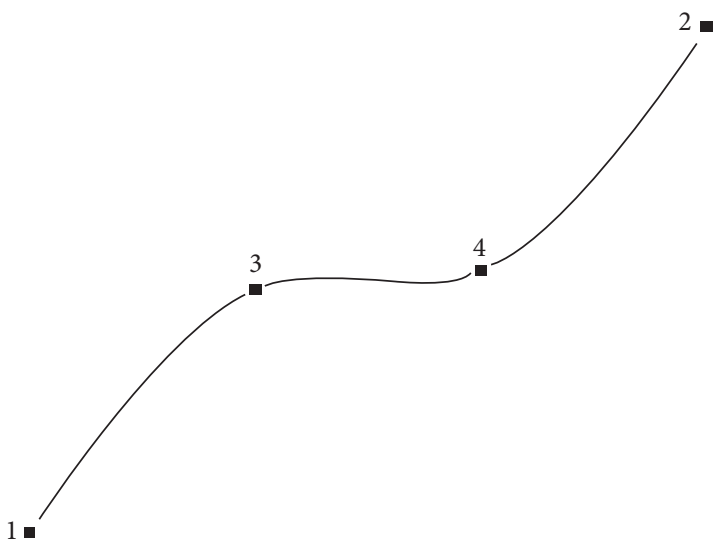

FIGURE 3: The cubed curve for integration.

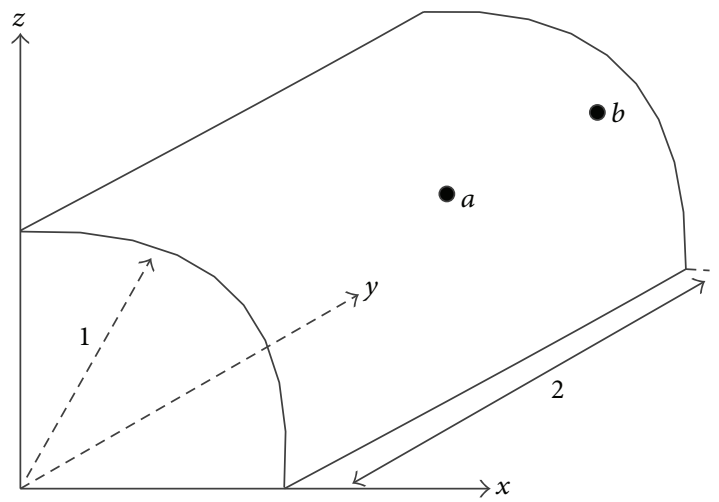

FIgURE 4: The cylindrical surface.

Using the derived analytical expressions in this paper, the third-order derivatives can be obtained:

$$
\begin{aligned}
& \frac{\partial^{3} x_{2}^{\prime}}{\partial x_{1}^{\prime 3}}=-6.0000, \\
& \frac{\partial^{3} \xi}{\partial x_{1}^{\prime 3}}=-6.7493 \times 10^{-6} .
\end{aligned}
$$

The results of the CVDM are exactly the same with that in (53). It can be seen that the CVDM and the analytical expressions in $(7 c)-(8 c)$ are validated by each other.

Secondly, the singular integral is evaluated using the present method. The result is 0.229935 , with the Gauss order being 6 .

This integral is also evaluated using the Gauss quadrature rule for logarithmic function [12]. The result is $J=0.226920$. It can be seen that the present method is effective and with high accuracy, for higher order curved boundary.

3.3. A Supersingular Integral over a Curved Surface. In this example, the accuracy of the present method in handling singular integrals over a highly curved surface is examined. The boundary element is a cylindrical surface with radius 1 and the rotational angle is $90^{\circ}$. The geometrical dimensions are shown in Figure 4.
TABLE 2: Results for two points $a$ and $b(\beta=3)$.

\begin{tabular}{lcc}
\hline & Point $a$ & Point $b$ \\
\hline Method in [14] & -0.3438 & -0.4971 \\
Present method & -0.3458 & -0.4981 \\
\hline
\end{tabular}

The boundary integral is as follows:

$$
I\left(x^{p}\right)=\int_{\Gamma} \frac{-1}{4 \pi r^{\beta}}\left[3 r_{, 3} \frac{\partial r}{\partial n}-n_{3}\right] d \Gamma .
$$

Two source points, $a$ and $b$, are located at positions with intrinsic coordinates of $(0,0)$ and $(0.66,0)$.

Guiggiani et al. [14] computed this integral for the case of $\beta=3$. Table 2 lists the results using both methods. For the present method, the Gauss order is 4 .

From Table 2, it can be seen that he results using the present method agree well with the Guiggiani et al.'s results, which indicate the high accuracy of the present method for singular integrals over a highly curved surface.

\section{Conclusion and Future Work}

In the present work, high order projection plane method for evaluation of supersingular curved boundary integrals in BEM has been developed, by considering the thirdorder derivatives when expanding the geometry quantities at the field point as Taylor series. Corresponding analytical expressions are derived, and unified expressions are obtained for both two-dimensional and three-dimensional problems.

The present method extends the application range of the method in [11]. That is, the singular integrals over higher order elements, such as cubed curve, can be directly evaluated. The complex-variable-differentiation method is introduced to determine the third-order derivatives, which effectively avoids rather complicated analytical derivations for the three-dimensional analysis. For the two-dimensional boundary integral, the third-order derivatives are analytically derived and calculated, which are used to verify the complexvariable-differentiation method.

The results show that the present method is effective and with high accuracy for high order singular curved boundary integrals.

Future work is to apply the mathematical derivations and equations to evaluate the singularities involved in radial integration boundary element method in solving nonhomogeneous thermoelastic problems.

\section{Conflict of Interests}

The authors declare that there is no conflict of interests regarding the publication of this paper.

\section{Acknowledgments}

The authors gratefully acknowledge the National Natural Science Foundation of China (51576026 and 11572075) and 
the Fundamental Research Funds for the Central Universities (DUT14LK03).

\section{References}

[1] C. F. Rowlatt and T. N. Phillips, "A spectral element formulation of the immersed boundary method for Newtonian fluids," Computer Methods in Applied Mechanics and Engineering, vol. 298, pp. 29-57, 2016.

[2] H. Qi, L. M. Ruan, H. C. Zhang, Y. M. Wang, and H. P. Tan, "Inverse radiation analysis of a one-dimensional participating slab by stochastic particle swarm optimizer algorithm," International Journal of Thermal Sciences, vol. 46, no. 7, pp. 649-661, 2007.

[3] S. Zhang, Y. Zhu, B. Qiao, and Z. Zhang, "System model of heat and mass transfer process for mobile solvent vapor phase drying equipment," Mathematical Problems in Engineering, vol. 2014, Article ID 267276, 11 pages, 2014.

[4] T. Lu, W. W. Han, P. X. Jiang, Y. H. Zhu, J. Wu, and C. L. Liu, "A two-dimensional inverse heat conduction problem for simultaneous estimation of heat convection coefficient, fluid temperature and wall temperature on the inner wall of a pipeline," Progress in Nuclear Energy, vol. 81, pp. 161-168, 2015.

[5] H. Qi, Y. B. Qiao, S. C. Sun, Y. Yao, and L. Ruan, "Image reconstruction of two-dimensional highly scattering inhomogeneous medium using MAP-based estimation," Mathematical Problems in Engineering, vol. 2015, Article ID 412315, 9 pages, 2015.

[6] J. Sladek, V. Sladek, and S. N. Atluri, "Local boundary integral equation (LBIE) method for solving problems of elasticity with nonhomogeneous material properties," Computational Mechanics, vol. 24, no. 6, pp. 456-462, 2000.

[7] J. T. Chen and H.-K. Hong, "Review of dual boundary element methods with emphasis on hypersingular integrals and divergent series," Applied Mechanics Reviews, vol. 52, no. 1, pp. 17-33, 1999.

[8] Ch. Zhang, M. Cui, J. Wang, X. W. Gao, J. Sladek, and V. Sladek, "3D crack analysis in functionally graded materials," Engineering Fracture Mechanics, vol. 78, no. 3, pp. 585-604, 2011.

[9] Y. Ochiai, "Three-dimensional thermo-elastoplastic analysis by tripe-reciprocity boundary element method," Engineering Analysis with Boundary Elements, vol. 35, pp. 478-488, 2011.

[10] J. Bremer and Z. Gimbutas, "On the numerical evaluation of the singular integrals of scattering theory," Journal of Computational Physics, vol. 251, pp. 327-343, 2013.

[11] X.-W. Gao, W.-Z. Feng, K. Yang, and M. Cui, "Projection plane method for evaluation of arbitrary high order singular boundary integrals," Engineering Analysis with Boundary Elements, vol. 50, pp. 265-274, 2015.

[12] J. L. Tsalamengas, "Quadrature rules for weakly singular, strongly singular, and hypersingular integrals in boundary integral equation methods," Journal of Computational Physics, vol. 303, pp. 498-513, 2015.

[13] V. Sladek and J. Sladek, Singular Integrals in Boundary Element Methods, WIT Press, Southampton, UK, 1998.

[14] M. Guiggiani, G. Krishnasamy, T. J. Rudolphi, and F. J. Rizzo, “A general algorithm for the numerical solution of hypersingular boundary integral equations," Journal of Applied Mechanics, vol. 59, no. 3, pp. 604-614, 1992.

[15] P. Bettini, M. F. Palumbo, and R. Specogna, "A boundary element method for eddy-current problems in fusion devices," Fusion Engineering and Design, vol. 96-97, pp. 620-623, 2015.
[16] A. Frangi and M. Guiggiani, "Direct approach for boundary integral equations with high-order singularities," International Journal for Numerical Methods in Engineering, vol. 49, no. 7, pp. 871-898, 2000.

[17] S. Mukherjee and Y. X. Mukherjee, "The hypersingular boundary contour method for three-dimensional linear elasticity," Journal of Applied Mechanics, vol. 65, no. 2, pp. 300-309, 1998.

[18] W. Chen, J.-Y. Zhang, and Z.-J. Fu, "Singular boundary method for modified Helmholtz equations," Engineering Analysis with Boundary Elements, vol. 44, pp. 112-119, 2014.

[19] M. H. Bazyar and A. Talebi, "Scaled boundary finite-element method for solving non-homogeneous anisotropic heat conduction problems," Applied Mathematical Modelling, vol. 39, no. 23-24, pp. 7583-7599, 2015.

[20] J. N. Lyness and C. B. Moler, "Numerical differentiation of analytic functions," SIAM Journal on Numerical Analysis, vol. 4, pp. 202-210, 1967.

[21] M. Cui, X. Gao, and H. Chen, "Inverse radiation analysis in an absorbing, emitting and non-gray participating medium," International Journal of Thermal Sciences, vol. 50, no. 6, pp. 898905, 2011.

[22] M. Cui, X. W. Gao, and H. G. Chen, "A new approach for the equivalent gray radiative property of a non-gray medium using a modified zonal method and the complex-variabledifferentiation method," Journal of Quantitative Spectroscopy and Radiative Transfer, vol. 112, no. 8, pp. 1336-1342, 2011.

[23] M. Cui, Q. H. Zhu, and X. W. Gao, "A modified conjugate gradient method for transient nonlinear inverse heat conduction problems: a case study for identifying temperature-dependent thermal conductivities," ASME Journal of Heat Transfer, vol. 136, no. 9, Article ID 091301, 2014.

[24] M. Cui, W.-W. Duan, and X.-W. Gao, "A new inverse analysis method based on a relaxation factor optimization technique for solving transient nonlinear inverse heat conduction problems," International Journal of Heat and Mass Transfer, vol. 90, pp. 491498, 2015. 


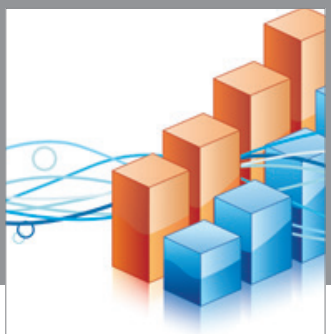

Advances in

Operations Research

vatem alat4

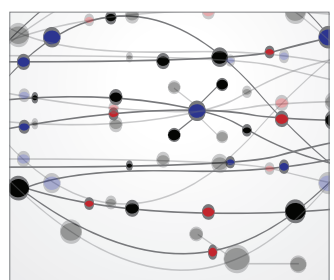

\section{The Scientific} World Journal
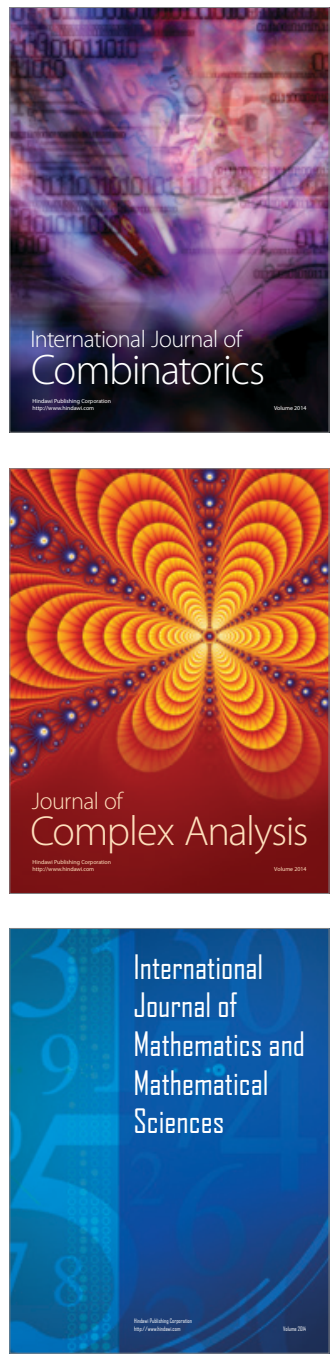
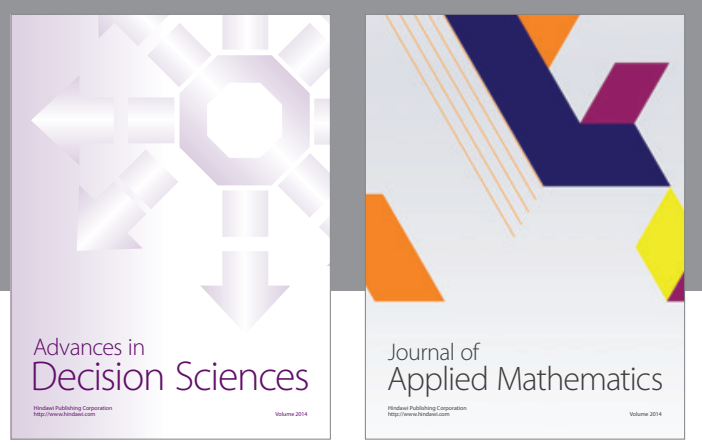

Algebra

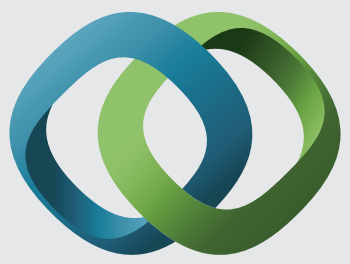

\section{Hindawi}

Submit your manuscripts at

http://www.hindawi.com
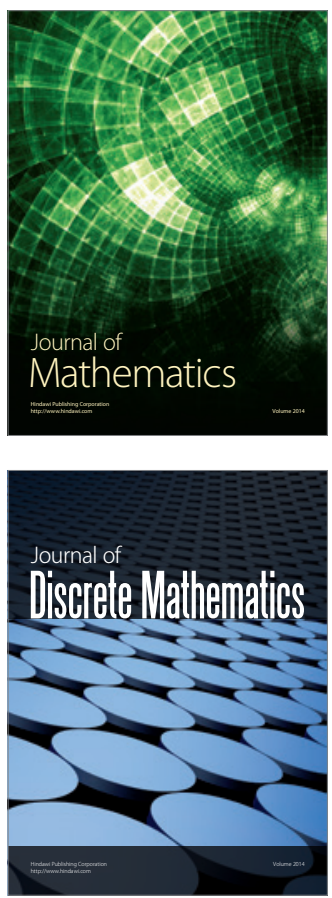

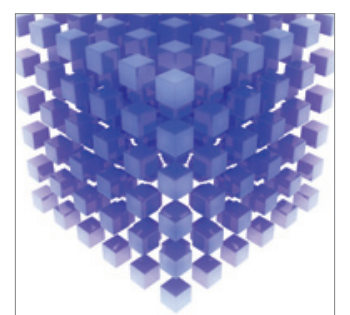

Mathematical Problems in Engineering
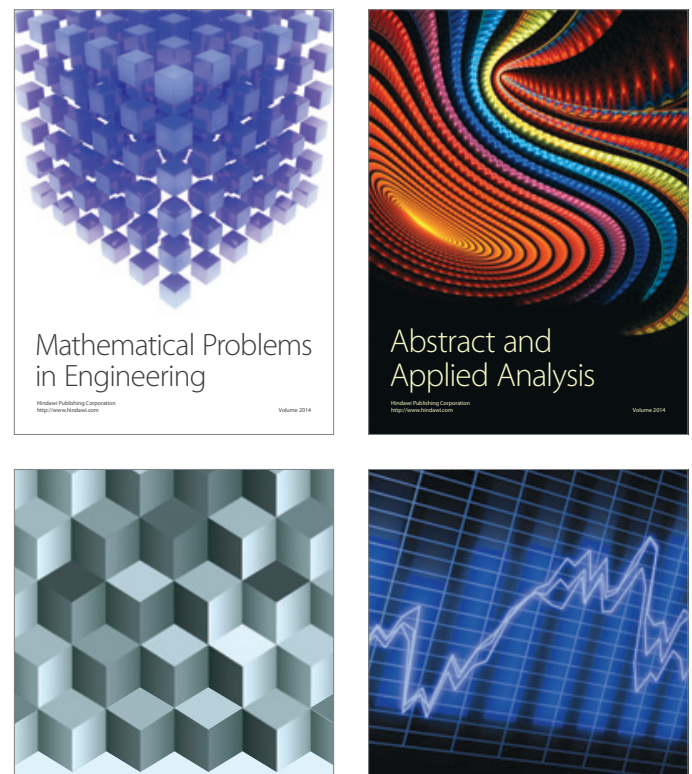

Journal of

Function Spaces

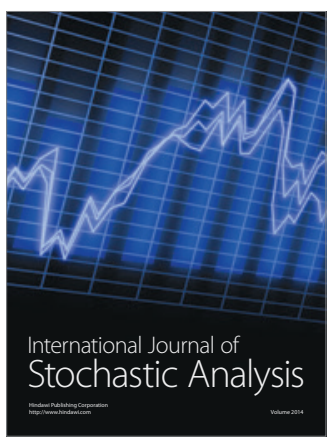

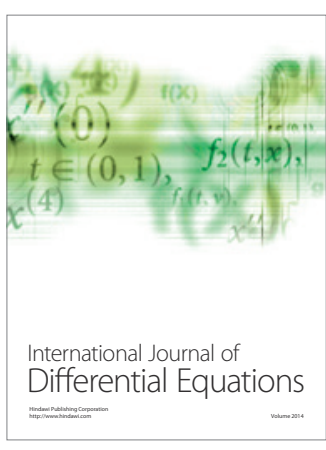
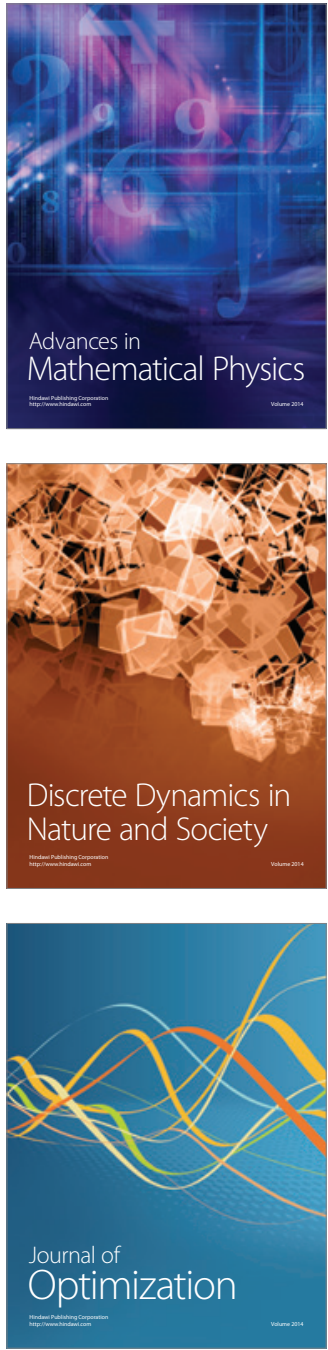